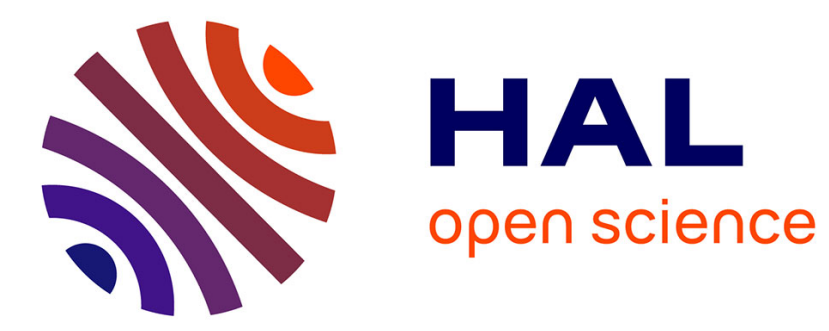

\title{
Category "viewpoints and debates" Is trastuzumab as a single agent obsolete in early breast cancer? Yes
}

\author{
Hervé Bonnefoi, Camille Chakiba
}

\section{To cite this version:}

Hervé Bonnefoi, Camille Chakiba. Category "viewpoints and debates" Is trastuzumab as a single agent obsolete in early breast cancer? Yes. Breast, 2019, 43, pp.146 - 148. 10.1016/j.breast.2018.10.008 . hal-03485704

\author{
HAL Id: hal-03485704 \\ https://hal.science/hal-03485704
}

Submitted on 20 Dec 2021

HAL is a multi-disciplinary open access archive for the deposit and dissemination of scientific research documents, whether they are published or not. The documents may come from teaching and research institutions in France or abroad, or from public or private research centers.
L'archive ouverte pluridisciplinaire HAL, est destinée au dépôt et à la diffusion de documents scientifiques de niveau recherche, publiés ou non, émanant des établissements d'enseignement et de recherche français ou étrangers, des laboratoires publics ou privés.

\section{(ㄷ)(1) $\$$}

Distributed under a Creative Commons Attribution - NonCommerciall 4.0 International 


\title{
Category "viewpoints and debates"
}

Is trastuzumab as a single agent obsolete in early breast cancer? Yes.

\author{
Hervé Bonnefoi (1) \\ Camille Chakiba (1)
}

(1) Department of Medical Oncology, Institut Bergonié Unicancer, Univ. Bordeaux, INSERM U1218, INSERM CIC1401, Bordeaux, France

\section{Corresponding author}

Prof Hervé Bonnefoi

Department of Medical Oncology, Institut Bergonié Unicancer, Univ. Bordeaux, INSERM U1218, INSERM CIC1401, 229 Cours de l'Argonne, F-33000 Bordeaux, France.

Email: h.bonnefoi@bordeaux.unicancer.fr

Phone: +33 556333269 Fax: +33 556333330 


\section{Summary (250 words max)}

In 2005, two adjuvant trials revolutionised breast cancer treatment demonstrating a $50 \%$ reduction in relapses when trastuzumab was added to adjuvant chemotherapy. In order to improve further on these results three large phase III trials were conducted. ALTTO trial evaluated lapatinib. This trial was negative and lapatinib was quite toxic. ExteNET trial evaluated neratinib in patients who already had completed adjuvant treatment with trastuzumab. Neratinib reduced the risk of relapse by $27 \%$ and the drug is FDA approved. However $40 \%$ of patients experienced grade 3 diarrhoea and this toxicity profile will be an issue in daily practice. APHINITY trial evaluated a combination of pertuzumab and trastuzumab. Pertuzumab reduced the risk of relapse by $19 \%$ with a good toxicity profile. However the absolute invasive disease-free survival (IDFS) benefit at 4 years was only $1.7 \%$. Despite this modest absolute benefit we believe that pertuzumab should be added to trastuzumab at least in two indications: first, in patients with node positive breast cancer, in whom the absolute IDFS benefit is $3.2 \%$ and qualify for a high clinical benefit based on ESMO magnitude of clinical benefit scale; second, in patients suitable for neoadjuvant chemotherapy. In this group, the absolute IDFS benefit could be estimated as high as $7 \%$ (starting pertuzumab in the neoadjuvant setting and following it in the adjuvant setting up to a total of 18 injections). Our arguments are developed in this viewpoint. Pertuzumab is approved in these two indications by FDA and EMA. 


\section{Introduction}

In 2005, the results of US joint analysis and HERA trial presented at ASCO revolutionised breast cancer treatment. Both studies demonstrated a $50 \%$ reduction in relapses when trastuzumab was added for one year to adjuvant chemotherapy [1, 2]. This reduction translated into an absolute disease-free survival (DFS) benefit of $12 \%$ at 3 years (75.4$87.1 \%$ ) in the US joint analysis [1] and 8.4\% at 2 years (77.4-85.8\%) in the HERA trial [2]. These large absolute DFS benefits continued $(11.5 \%$ in the US joint analysis and $6.8 \%$ in the HERA trial) in long term follow-up with an absolute overall survival gains of $8.8 \%$ and $6.5 \%$, respectively [3, 4]. However, despite this dramatic improvement with trastuzumab, approximately one-third of the patients will still relapse and eventually die of their disease [3, 4].

\section{Phase III trials evaluating dual anti-HER2 blockade}

Consequently, three phase III trials evaluating new anti-human epidermal growth factor receptor 2 (HER2) treatments were conducted in the adjuvant setting: ALTTO, ExteNET and APHINITY. In the first two trials, oral lapatinib and neratinib (anti-HER2 and anti-HER1 tyrosine kinase inhibitors, respectively) were tested. In the third trial, pertuzumab, a monoclonal antibody that inhibits HER2 heterodimerisation with other HER family receptors was combined with trastuzumab.

The ALTTO trial compared four anti-HER2 treatments over one year: trastuzumab in arm A, lapatinib in arm B, trastuzumab for 3 months followed by lapatinib in arm C and trastuzumab plus lapatinib (combined treatment) in arm $D$ [5]. The primary endpoint was invasive disease-free survival (IDFS). Arm B was stopped early for futility to demonstrate non-inferiority. Of the two comparisons planned, the first one (arm C vs arm A) failed to demonstrate non-inferiority. The second comparison (arm D vs arm A) demonstrated a 16\% reduction in the risk of relapse (HR $0.84 ; 95 \% \mathrm{Cl}: 0.70-1.02 ; \mathrm{P}=0.048)$ but was statistically not significant at the pre-specified $P$ value $\leq 0.025$. Lastly, the combined treatment was more toxic. The most frequent grade 3-4 toxicities were diarrhoea in 15\%, rash in 5\% and liver toxicity in $3 \%$ of patients. Based on these data lapatinib was not approved in early breast cancer.

In ExteNET trial, 2840 patients who completed neoadjuvant or adjuvant chemotherapy plus trastuzumab were randomised to receive either neratinib or placebo for one year. The primary endpoint analysis (IDFS at 2 years) was positive [6]. A second analysis was performed with a median follow-up of 5.2 years [7]. Less invasive relapses were observed in the neratinib arm (HR 0.73; 95\% Cl: 0.57-0.92; $\mathrm{p}=0.0083$ ). This difference was mainly observed in the hormone-receptor-positive group. Grade 3 diarrhoea was observed in $40 \%$ of patients (one patient experienced a grade 4). Despite its toxicity profile, neratinib was 
approved by the Food and Drug Administration (FDA) both for hormone-receptor-positive or negative tumours [8] and the European Medicines Agency (EMA) expressed a positive opinion in patients with hormone-receptor-positive tumours only [9]. However, we believe that grade 3 diarrhoea will be an issue in daily practice.

In the APHINITY trial, 4805 patients were randomised after surgery to receive either one year of pertuzumab or placebo in addition to standard adjuvant chemotherapy plus one year of adjuvant trastuzumab [10]. The relative risk reduction (RRR) of invasive relapse with the addition of pertuzumab in APHINITY was $19 \%$ (HR 0.81; 95\%Cl: $0.66-1.00 ; P=0.045$ ) with a median follow-up of 45.4 months. Pertuzumab was very well tolerated. Grade 3 diarrhoea was observed in $9.8 \%$ of patients in the pertuzumab arm and $3.7 \%$ in the placebo arm. Cardiac toxicity did not increase. The absolute IDFS benefit at 3 years and 4 years was $0.9 \%$ and $1.7 \%$, respectively.

\section{Indications for pertuzumab in two high risk populations}

Some clinicians may argue that these the APHINITY results do not support the use of pertuzumab in early breast cancer. We obviously agree with these colleagues when considering patients who has had surgery for a small node negative tumour and whose prognosis is excellent with weekly paclitaxel and trastuzumab single agent as demonstrated by Tolaney and colleagues [11]. We think the exact opposite and However we believe that pertuzumab should be added to trastuzumab in at least two indications: patients with node positive $\left(\mathrm{N}_{+}\right)$breast cancer and patients suitable for neoadjuvant chemotherapy.

First, we will focus on $3005 \mathrm{~N}+$ disease patients randomised in APHINITY trial. In the trastuzumab and pertuzumab arm, the RRR of relapse was $23 \%$ (HR $0.77 ; 95 \% \mathrm{Cl}$ : $0.62-$ $0.96 ; P=0.02)$. This $R R R$ translated to a $3.2 \%$ absolute gain at 4 years with very little additional toxicity.

In general, when assessing the potential value of a new treatment, we use the term "clinical benefit (CB)", a necessary balance between efficacy (more precisely, the absolute magnitude of the benefit) and toxicity. In patients with $\mathrm{N}+$ disease, CB observed with the addition of pertuzumab may be interpreted by many clinicians as relevant while others may consider it as modest or futile. In order to avoid a subjective interpretation of the term CB, we should refer to the European Society of Medical Oncology (ESMO) magnitude of clinical benefit scale (MCBS) [12]. The basic principle of this scale is that the CB of a new treatment for a patient is either to live longer or to live better [12]. ESMO experts consider DFS as a good surrogate for survival in studies with curative intent but without mature survival data. In a curative setting, the scale includes three grades: A, B or C. Grades correspond to an improvement in DFS alone and are defined using HR threshold (by definition in ESMO- 
MCBS, this HR threshold refers to the lower limit of the $95 \% \mathrm{Cl}$ ). Grade A corresponds to an improvement in DFS alone with a $H R<0.65$, grade $B$ with a HR 0.65-0.8 and grade $C$ with a $H R>0.8$. Of note, pre-planned subgroup analysis can be scored separately, which applies to the $\mathrm{N}+$ subgroup in APHINITY trial. Thus based on the ESMO-MCBS, CB of APHINTY in the $\mathrm{N}+$ subgroup is scaled $\mathrm{A}$ (as mentioned above, the lower limit of the $95 \% \mathrm{Cl}$ is 0.62 ) which means for ESMO experts "a high level of CB" [12].

Despite this ESMO-MCBS grade A, some clinicians still believe that a $3 \%$ absolute decrease in recurrence is very modest. However, it is very likely that the same clinicians are using taxanes as standard treatment for $\mathrm{N}$ + breast cancer. It is worth reminding them that the absolute benefit of taxanes is very similar to the one observed with pertuzumab in $\mathrm{N}+$ breast cancer patients. The use of taxanes in the adjuvant setting results in an absolute decrease of the recurrence rate at 5 years of only $2.9 \%$ with an RRR of $14 \%$ (HR 0.86; 95\% Cl: $0.82-$ $0.91 ; \mathrm{P}<0.00001)$ [13]. These data are from 33000 patients $\left(82 \% \mathrm{~N}_{+}\right)$included in trials comparing anthracycline with and without taxanes (same treatment duration in both groups).

The second indication of pertuzumab should be in the neoadjuvant setting both concomitantly with chemotherapy and after surgery in the adjuvant setting. As mentioned above, in patients with $\mathrm{N}+$ breast cancer, the addition of pertuzumab translated to a $3.2 \%$ absolute gain at 4 years. In patients suitable for neoadjuvant chemotherapy the risk of relapse is higher. The absolute gain is likely to be much more prominent in this group as higher the risk, higher the gain. For a precise estimation, we use the survival data of patients treated with chemotherapy and trastuzumab only in the standard arm of NeoALTTO which were recently updated with a median follow-up of 6.7 years [14]. Of note, the population of patients included in NeoALTTO cannot be considered as particularly high risk compared to other neoadjuvant trials (for example, $60 \%$ of patients were cT2). In this trial, $33 \%$ of patients presented with an invasive relapse $(29 \%$ and $37 \%$ in the hormone-receptor-positive and negative groups, respectively). If we focus on hormone-receptor-negative patients and apply the $19 \%$ RRR observed in the APHINITY trial (we will not use the $23 \%$ RRR observed in the $\mathrm{N}+$ group in order not to overestimate the treatment effect), the absolute benefit at 6 years is $7 \%$. This cannot be considered as marginal. The number to treat to avoid one relapse is 14 . In practice, we recommend starting pertuzumab with trastuzumab in the neoadjuvant setting. In Neosphere trial, the pathological complete response rate almost doubled (from $21.5 \%$ to $39.3 \%$ ) when pertuzumab was added to trastuzumab and docetaxel in the neoadjuvant setting [15]. We also recommend following it up with adjuvant pertuzumab plus trastuzumab, up to 18 injections (including the neoadjuvantly administered injections). Of note, the effect of a shorter duration of this dual blockade has not been evaluated in a phase III trial. 
Both the abovementioned indications for pertuzumab in two high-risk of relapse populations are approved by FDA and EMA. In 2013, the FDA granted accelerated approval to pertuzumab as a neoadjuvant treatment [16]. This approval was mainly based on the results of the Neosphere trial [15]. It should be reminded that that approval was conditional depending on the results of the APHINITY trial looking at difference in DFS as a primary endpoint. In 2017, the results of APHINITY demonstrated a survival gain and therefore the FDA granted a regular approval for pertuzumab both "as neoadjuvant treatment of patients with HER2-positive, locally advanced, inflammatory in patients with locally-advanced, inflammatory, or early stage breast cancer in combination with trastuzumab and chemotherapy and in patients with early breast cancer (either greater than $2 \mathrm{~cm}$ in diameter or node positive) as part of a complete treatment regimen for early breast cancer" [17]. The EMA also approved pertuzumab as neoadjuvant treatment and extended the indication recently to the adjuvant setting in patients "at high risk of recurrence" [18].

\section{Issues in Europe}

However, in Europe, there exist three issues. First, there is already a big heterogeneity between countries as far as access to neoadjuvant pertuzumab (in general from 3 to 4 injections) is concerned. Second, the incremental cost of delivering a total of eighteen injections of pertuzumab "as part of a complete treatment regimen for early breast cancer" (approximately 70'000 euros per patient) is an issue for reimbursement by national health systems. In addition, there data on cost-effectiveness are lacking. Third, and second, different interpretations of the term "high risk" can be interpreted in different ways. Methods assessing the risk of recurrence in patients treated with standard treatment and trastuzumab as a single agent only may help. Composite predictors including clinicopathological parameters and tumour markers similar to the ones developed in patients with hormonereceptor-positive and HER2-negative tumours [19, 20] should also be developed in HER2positive breast cancer patients. As HER2-positive hormone-receptor-positive and negative tumours have different relapse patterns over time, different predictors need to be developed [21]. These predictors may allow assessing the risk for an individual patient and potentially to better estimate the absolute DFS benefit with dual HER2-blockade.

\section{Conclusion}

The results of adjuvant trials with trastuzumab in early breast cancer were a revolution. Pertuzumab is an evolution. The abovementioned risk of recurrence predictors should help to refine the indications for pertuzumab in high-risk of relapse populations and should reduce the economic toxicity of dual anti-HER2 therapy. Until we get these predictors we have to take our responsibilities as clinicians in front of our patients based on published data. 


\section{Acknowledgements}

We thank Ravi Nookala of Institut Bergonié for the medical writing service.

1. Romond $\mathrm{EH}$, Perez EA, Bryant $\mathrm{J}$ et al. Trastuzumab plus adjuvant chemotherapy for operable HER2-positive breast cancer. N Engl J Med 2005; 353: 1673-1684.

2. Piccart-Gebhart MJ, Procter M, Leyland-Jones B et al. Trastuzumab after adjuvant chemotherapy in HER2-positive breast cancer.[see comment]. New England Journal of Medicine 2005; 353: 1659-1672.

3. Cameron D, Piccart-Gebhart MJ, Gelber RD et al. 11 years' follow-up of trastuzumab after adjuvant chemotherapy in HER2-positive early breast cancer: final analysis of the HERceptin Adjuvant (HERA) trial. Lancet 2017; 389: 1195-1205.

4. Perez EA, Romond EH, Suman VJ et al. Trastuzumab plus adjuvant chemotherapy for human epidermal growth factor receptor 2-positive breast cancer: planned joint analysis of overall survival from NSABP B-31 and NCCTG N9831. J Clin Oncol 2014; 32: 3744-3752. 5. Piccart-Gebhart M, Holmes E, Baselga $J$ et al. Adjuvant Lapatinib and Trastuzumab for Early Human Epidermal Growth Factor Receptor 2-Positive Breast Cancer: Results From the Randomized Phase III Adjuvant Lapatinib and/or Trastuzumab Treatment Optimization Trial. J Clin Oncol 2016; 34: 1034-1042.

6. Chan A, Delaloge S, Holmes FA et al. Neratinib after trastuzumab-based adjuvant therapy in patients with HER2-positive breast cancer (ExteNET): a multicentre, randomised, double-blind, placebo-controlled, phase 3 trial. Lancet Oncol 2016; 17: 367-377.

7. Martin M, Holmes FA, Ejlertsen B et al. Neratinib after trastuzumab-based adjuvant therapy in HER2-positive breast cancer (ExteNET): 5-year analysis of a randomised, doubleblind, placebo-controlled, phase 3 trial. Lancet Oncol 2017; 18: 1688-1700.

8. US Food and Drug Administration. Prescribing Information for Neratinib. N. https://http://www.accessdata.fda.gov/drugsatfda_docs/label/2017/208051s000lbl.pdf

9. European Medicines Agency. Positive opinion. http://www.ema.europa.eu/docs/en_GB/document_library/Summary_of_opinion_Initial_authorisation/human/004030/WC500244282.pdf N.

10. von Minckwitz G, Procter M, de Azambuja E et al. Adjuvant Pertuzumab and Trastuzumab in Early HER2-Positive Breast Cancer. N Engl J Med 2017; 377: 122-131. 11. Tolaney SM, Barry WT, Dang CT et al. Adjuvant paclitaxel and trastuzumab for nodenegative, HER2-positive breast cancer. N Engl J Med 2015; 372: 134-141.

12. Cherny NI, Sullivan R, Dafni U et al. A standardised, generic, validated approach to stratify the magnitude of clinical benefit that can be anticipated from anti-cancer therapies: the European Society for Medical Oncology Magnitude of Clinical Benefit Scale (ESMOMCBS). Ann Oncol 2015; 26: 1547-1573.

13. EBCTCG. Comparisons between different polychemotherapy regimens for early breast cancer: meta-analyses of long-term outcome among 100,000 women in 123 randomised trials. Lancet 2012; 379: 432-444.

14. Huober J, McCormick E, Fugamelli D et al. Survival outcomes of the NeoALTTO study: update results of a randomized multicenter phase III neoadjuvant trial. J Clin Oncol 2017; 35: (suppl; abstr 512).

15. Gianni L, Pienkowski T, Im YH et al. Efficacy and safety of neoadjuvant pertuzumab and trastuzumab in women with locally advanced, inflammatory, or early HER2-positive 
breast cancer (NeoSphere): a randomised multicentre, open-label, phase 2 trial. Lancet Oncol 2012; 13: 25-32.

16. Amiri-Kordestani L, Wedam S, Zhang $L$ et al. First FDA approval of neoadjuvant therapy for breast cancer: pertuzumab for the treatment of patients with HER2-positive breast cancer. Clin Cancer Res 2014; 20: 5359-5364.

17. US Food and Drug Administration. Prescribing Information for Perjeta. I.pdf. https://http://www.accessdata.fda.gov/drugsatfda_docs/label/2017/125409s113s118lb

18. European Medicines Agency. Summary of Product Characteristics for Perjeta.

http://www.ema.europa.eu/ema/index.jsp?curl=pages/medicines/human/medicines/00 2547/human_med_001628.jsp\&mid=WC0b01ac058001d124 pE.

19. Viale $\bar{G}$, Regan MM, Dell'Orto $P$ et al. Which patients benefit most from adjuvant aromatase inhibitors? Results using a composite measure of prognostic risk in the BIG 1-98 randomized trial. Ann Oncol 2011; 22: 2201-2207.

20. Regan MM, Francis PA, Pagani O et al. Absolute Benefit of Adjuvant Endocrine Therapies for Premenopausal Women With Hormone Receptor-Positive, Human Epidermal Growth Factor Receptor 2-Negative Early Breast Cancer: TEXT and SOFT Trials. J Clin Oncol 2016; 34: 2221-2231.

21. Untch M, Gelber RD, Jackisch $\mathrm{C}$ et al. Estimating the magnitude of trastuzumab effects within patient subgroups in the HERA trial. Ann Oncol 2008; 19: 1090-1096. 